\title{
Plasma Near A Divertor Plate: Effects of Surface Roughness and Particle Drifts
}

R. H. Cohen and D. D. Ryutov

This article was submitted to International Workshop on Plasma Edge Theory, Tajimi, Japan, October 4-6, 1999

U.S. Department of Energy

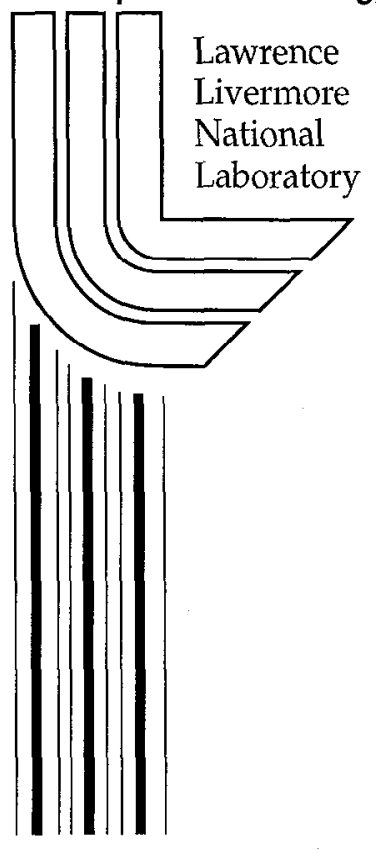

\section{September 30, 1999}





\section{DISCLAIMER}

This document was prepared as an account of work sponsored by an agency of the United States Government. Neither the United States Government nor the University of California nor any of their employees, makes any warranty, express or implied, or assumes any legal liability or responsibility for the accuracy, completeness, or usefulness of any information, apparatus, product, or process disclosed, or represents that its use would not infringe privately owned rights. Reference herein to any specific commercial product, process, or service by trade name, trademark, manufacturer, or otherwise, does not necessarily constitute or imply its endorsement, recommendation, or favoring by the United States Government or the University of California. The views and opinions of authors expressed herein do not necessarily state or reflect those of the United States Government or the University of California, and shall not be used for advertising or product endorsement purposes.

This is a preprint of a paper intended for publication in a journal or proceedings. Since changes may be made before publication, this preprint is made available with the understanding that it will not be cited or reproduced without the permission of the author.

This report has been reproduced

directly from the best available copy.

Available to DOE and DOE contractors from the

Office of Scientific and Technical Information

P.O. Box 62, Oak Ridge, TN 37831

Prices available from (423) 576-8401

http://apollo.osti.gov/bridge/

Available to the public from the

National Technical Information Service

U.S. Department of Commerce

5285 Port Royal Rd.,

Springfield, VA 22161

http://www.ntis.gov/

OR

Lawrence Livermore National Laboratory

Technical Information Department's Digital Library

http://www.llnl.gov/tid/Library.html 


\title{
Plasma near a divertor plate: effects of surface roughness and particle drifts
}

\author{
R.H. Cohen, D.D. Ryutov \\ Lawrence Livermore National Laboratory, Livermore, CA 94551, USA
}

\begin{abstract}
The surface of a divertor plate has usually a certain degree of "roughness." Depending of the material and the exposure time, the size of surface features may range from submicrons to a fraction of a millimeter, covering a range of spatial scales from well below the electron gyroradius, $\rho_{e}$, to significantly above the ion gyroradius, $\rho_{i}$. The plasma approaches the divertor plate along a magnetic field which forms a shallow angle, $\alpha<<1$, with the plate surface. Under such circumstances, a significant "shadowing" effect takes place, with only a small part of the surface being accessible to the plasma particles.

A methodology is presented that allows one to find the fraction $\left(\varepsilon_{e}\right.$ and $\left.\varepsilon_{i}\right)$ of the surface -geometrically accessible for the electrons and the ions. At small $\alpha, \varepsilon_{e, i}$ are typically small, meaning a strong local wharicement of heat and particle fluxes. In a broad range of parameters, $\varepsilon_{\varepsilon}$ is also auch smaller than $\varepsilon_{i}$ As the surface features are usually greater than the Debye radius, this leads to the formation of an ambipolar potential which causes reflection of part of the ions from the surface. The resulting albedo of the divertor plate for the plasma ions can be as high as $50 \%$. Gradual diffusion of the plasma electrons into the zones rellecting the ions, reduces ambipolar fields and brings the ion albedo back to very small values. We present estimates of the time scales governing the neutralization process. This time scale shows itself up in the sheath boundary conditions for non-steady-state perturbations.

The effect of surface roughness on secondary electron emission is discussed and it is shown that, depending on the surface structure, it may be smaller or greater than for a perfectly flat plate.

We consider modifications of the sheath current-voltage characteristics by particle drifts. The presence of reflected ions reduces the ion diamagnetic current in the ion sub-sheath and significantly changes the ion response. This, in turn, affects sheath-controlled instabilities, which are sensitive to the tilt of the magnetic field.
\end{abstract}

\section{Introduction}

During the last few, years, a considerable progress has been made in the analysis of plasmia interactions with non-planar surfacés in a tilted magnetic field [1-6]. The nonplanarity may rarige from a smooth waviness $[1,3,6]$, with the wavelengths exceeding the ion gyroradius by many orders of magnitude, to a small-scale "roughness", with sharp surface features having the size from a few ion gyroradii to less than electron gyroradius $[2,4-6]$. When the magnetic field intersects the "averaged" surface at a shallow angle, $\alpha \ll 1$, effects of non-planarity become particularly important because of a very pronounced "shadowing" effect comes up, making a part of the space near the surface inaccessible for one or both spieces of the incoming plasma flow. The objects where such phenomena may occur range from divertor plates of fusion devices $[4,5]$ to surfaces of large spacecraft at low Earth orbits [7].

In this paper we concentrate on the issues related to problems of rough surfaces, with the structures of the type shown in Fig. 1, in the parameter domain typical for the divertors of fusion devices. A general methodology for evaluating the geometrically accessible region for various relationships between the characteristic height, $h$, of the surface structures, and the particle gyroradii $\left(\rho_{i}\right.$ and $\left.\rho_{e}\right)$ has been developed in Refs. 4,5. 
For a magnetic field of 3T, and a deuterium plasma with a temperature of $T_{e} \sim T_{i} \equiv T \sim 50 \mathrm{eV}$, one has $\rho_{i} \sim 500 \mu \mathrm{m}$, and $\rho_{c} \sim 8 \mu \mathrm{m}$. Surface structures with the heights in this domain are quite typical for the divertor plates (see Refs.8-11).

In a broad range of heights one can meet the situation where a fraction of the surface geometrically accessible for the plasma electrons is much less than that geometrically accessible for the ions. By "geometrical accessibility" we mean constraints imposed by the equations of motions of single particles in a tilted magnetic field, without any account for possible ambipolarity constraints. Typically for the height $h$ of the surface bumps less than the ion gyroradius,

$$
\rho_{e}<h<\rho_{i}
$$

the electrons (which are more tightly bound to the magnetic field lines than the ions) penetrate much less deep into the shadows behind the peaks of the surface structures. The ions, which under condition (1) are essentially unmagnetized (see, however, Sec. 2 for some caveats), could penetrate much deeper into the shadows. However, as the plasma quasineutrality must be maintained, the ambipolar potential is formed near the surface of the zone accessibic for the electrons, and this potential repels the ions from the area inaccessible to electrons (Fig. 2). This, in turn, leads to formation of the complex potential structure both in the vicinity of the surface and in the bulk plasma. This also affects the heat load distribution and sputtering patterns of the rough surface.

The zones inaccessible for plasma electrons will be gradually filled by a cold neutralizing plasma thus making these zones again accessible to the plasma ions. Then, the fraction of the surface "wetted" by the plasma ions would become much greater than that wetted by the plasma electrons. The latter circumstance is important in evaluating the sputtering yield, the temperature distribution over the surface, and other phenomena occurring near the surface.

The question about the evolution of the surface "topography" under the action of the plasma flux does not have a universal answer and is not a subject of our paper. The problem we address is a plasma physics problem: given some structure is present, what are the consequences in terms of the plasma properties in the vicinity of the surface? By solving this part of the problem (in particular, by finding the fraction of the "wetted" surface), we provide the tools for studies of the processes occurring on a long time scale, such as the surface erosion.

The fact that divertor plate surfaces are generally rough, is well established. In some cases the roughness may be a mere reflection of the "grainy" structure of a plate material (some brands of graphite are an example). In other cases the source of roughness is a thermomechanical damage by short impulsive heat loads during disruptions, when the surface gets covered with ripples and droplets (for metallic plates), which solidify and then last for weeks under the normal operation scenario.

It is worth mentioning that it is not clear at all that the concentration of the heat flux near the tops of the bumps shouid necessarily lead to a smoothing of the surface (although, in some cases, especially in the case of refractory metals damaged by pulsed heat loads this scenario is possible). In fact, there are many other processes that play important role in , establishing a steady state surface relief: i) crack formation at the depth of a few grain size under the action of a cyclic heat load, with subsequent flaking; ii) cracking and blistering under the action of the absorbed neutral hydrogen; iii) blistering and flaking under the action of the neutron flux in the fusion reactor conditions; iv) damage from disruption events. The enhanced sputtering of the tops of the bumps is just one more factor that affects the evolution of the surface relief.

The structure of this paper is as follows: In Sec.2 (based on Ref. [12]) we describe particle trajectories over a flat surface in a tilted magnetic field. We demonstrate that either 
all the particles or a significant fraction of them approach the Debye sheath at a shallow angle $\sim \alpha^{1 / 2}$. This sets the stage for the interaction of particles with small-scale surface perturbations. In Sec. 3, we provide simple scaling equations allowing one to evaluate geometrically-accessible fraction of the surface for electrons and ions. In Sec. 4 we discuss new features arising from the quasineutrality constraint. In particular, we find that, in some regimes, $\sim 50 \%$ of the ions get reflected from the potential structures back into the plasma (even if the wall is made of the material perfectly absorbing ions). This affects both the hydrodynamic boundary condition and the sheath current-voltage characterisitics. In Sec. 5, we discuss various processes leading to a formation of neutralizing plasma and thereby restoring the geometrical accessibility. The time scale for the neutralization process can sometimes be as long as $10^{-4} \mathrm{~s}$ (the time comparable with the ion transit time over the connection length). Therefore, a new time-scale emerges in the problem of interaction of plasma perturbations with the wall (Sec. 6). In Sec. 7, we consider effect of the surface roughness on the secondary electron emission. Sec. 8 summarizes our results. gyroradius,

Throughout this paper we assume that the Debye radius is smaller than the electron

$$
\rho_{D}<\rho_{e}
$$

The wase where it is greater than $\rho_{e}$ (but sutil much less than $\rho_{i}$ ) can be considered in a similar fashion.

\section{Particle trajectories in a tilted magnetic field.}

Let us for a while neglect the presence of an ambipolar electric field outside the Debye sheath. Then the charged particles approaching the wall experience a simple spiral motion around the magnetic field lines which intersect the surface at a shallow angle. In this case, the ion approaching the wall along a strongly tilted magnetic field, when making one full gyrocircle, moves closer to the wall by only a small distance $\sim \alpha \rho_{i}$. The ion velocity components near the wall are:

$$
\mathrm{v}_{\mathrm{x}} \sim \mathrm{V}_{\mathrm{z}} \sim \mathrm{v}_{\mathrm{Ti}}, \mathrm{v}_{\mathrm{y}} \sim \mathrm{v}_{\mathrm{Ti}} \alpha^{1 / 2}
$$

(see Fig. 1 for the definition of the coordinate axes).The details of 'the corresponding analysis and the fuil ion distribution function near the wall can be found in Ref. 12. The projection of the typical ion trajectory on the divertor plate forms an angle $-45^{\circ}$ with the projection of the magnetic field.

Electric field present in the ion sub-sheath may also cause some changes of the overall picture in the case of non-uniformities which are smaller than the ion gyro-radius. As was shown in Ref. [12], some ions (predominantly those with a small energy) may be accelerated to the walls from the middle of the ion sub-sheath, and approach the wall at an angle $\sim 1$. They will be wetting the surface almost uniformly. However, as was shown in [12], the majority of the thermal ions will still approach the wall in the same qualitative way as at no electric field at all. One can describe them by Eq. (3).

\section{Particle absorption by a rough surface}

In this section we assume that the cold neutralizing plasma has already be formed in the "shadows", so that one can use a purely "geometrical" approach. 


\subsection{Ion absorption}

We first consider the case of very small $h$ 's, when the magnetic field doesn't directly affect the ion trajectories at a distance $\sim h$ from the wall. Even in this case, however, the presence of the magnetic field is important in that it causes the ions to approach the wall at a very shallow angle $\sim \alpha^{1 / 2}$ (see Sec. 2 ). To be able to apply our results for some other situations (see below) we consider a stream of ions with a density $n$ moving with a velocity $\mathrm{v}$ along straight trajectories forming some small angle $\beta$ (not necessarily $\beta \sim \alpha^{1 /}$ ) with the plane. The problem is: how far below the level of the mountain tops will the ions penetrate? This is a problem similar to the problem of a mountain range during sunrise: what fraction of the mountain land is illuminated? Let us denote by $\Delta h$ the corresponding distance from the mountain tops (here and below we mean by $\Delta h$ a characteristic, statistically averaged quantity). The surface area of the illuminated mountain top is $\sim \Delta h^{2}$. Each mountain top collects $n v \Delta h^{2}$ ions per unit time. As the number of the mountain tops per unit area of the mountain land is $\sim I / h^{2}$, the number of ions absorbed by the unit area of the plate per unit time is $\sim n v \Delta h^{2} / h^{2}$. On the other hand the number of the ions intersecting a unit surface clement parallel to the plate at some distance from the plate is $\beta n v$. Equating the two expressions, we obtain:

$$
\Delta h \sim \beta^{1 / 2} h
$$

Therefore,

As we have mentioned above, in the problem under consideration $\beta \sim \alpha^{1 / 2}$.

$$
\Delta h \sim \alpha^{1 / 4} h
$$

Accordingly, the fraction $\varepsilon_{i}$ of the surface wetted by the ions is:

$$
\varepsilon_{i} \sim \alpha^{1 / 2}
$$

Illuminated are south-east or north-east sides of the "mountains" (where field lines from the plasma in the sunrise analogy are assumed to intersect the east side), depending on whether the magnetic field is directed toward or away from the plate. Note, Eq. (6) remains formally valid even for infinitesimally small bumps (the constraints will be discussed in Sec. 4).

The estimate (5) is valid so long as the height $\Delta h$ is smaller than the distance $\alpha \rho_{i}$ by which the ion gyro-circle "descends" to the wall during one gyro-period (if $\Delta h$ becomes formally larger, the ion motion at the scales of interest begins to be affected by the magnetic field). Therefore, the applicability condition for the estimates (5), (6) reads:

$$
h \rho_{i} \alpha^{3 / 4}
$$

When it breaks down, the following approach [4] can be used. From the kinematics of the gyro-motion, it is clear that the characteristic vertical velocity of the ions whose gyro-centers have lowered by a distance $\Delta h$ since the moment when the gyro-circle has just touched the mountain tops (Fig. 3), is $\mathrm{v}_{\mathrm{Ti}}\left(\Delta h / \rho_{i}\right)^{1 / 2}$. The other two velocity components are of the order of $\mathrm{v}_{\mathrm{Ti}^{*}}$. The phase-space density of ions moving in a magnetic field is constant and, therefore, their volume density is proportional to the volume occupied in the velocity space. At distances exceeding $\rho_{i}+h$ from the wall, this volume is $\sim \mathrm{v}^{3} \mathrm{Ti}$, whereas in the layer of thickness $\Delta h$ containing the mountain tops it is $\sim\left(\Delta h / \rho_{i}\right)^{1 / 2} \mathrm{v}^{3}{ }_{\mathrm{T}}$. Accordingly, in the layer of thickness $\Delta h$, their density is $\sim n\left(\Delta h / \rho_{i}\right)^{I / 2}$. The number of ions absorbed by one mountain top per unit time is $\sim \Delta h^{2}\left(\Delta h / \rho_{i}\right)^{1 / 2} \mathrm{v}_{\mathrm{T} \text { i }}$, and the number of 
ions absorbed per unit area of the divertor plate per unit time is $\sim(\Delta h / h)^{2}\left(\Delta h / \rho_{i}\right)^{1 / 2} \mathbf{v}_{\mathrm{Ti}}$. On the other hand, the flux of ions approaching the divertor plate from the plasma is $\alpha n v_{\mathrm{Ti}}$ (per unit surface). Equating the two quantities, one finds that

$$
\Delta h / h \sim \alpha^{2 / 5}\left(\rho_{i} / h\right)^{1 / 5}
$$

The fraction of the surface wetted by the ions is:

$$
\varepsilon_{i} \sim\left(\frac{\Delta h}{h}\right)^{2} \sim \alpha^{4 / 5}\left(\frac{\rho_{i}}{h}\right)^{2 / 5} .
$$

The transition between the estimates (5) and (8) [and (6) and (9)] occurs at the point where inequality (7) becomes equality.

Equations (8) and (9) remain valid so long as the illuminated height $\Delta h$ remains smaller than $\rho_{i}$. This is so at

$$
h<\rho_{i} / \alpha^{1 / 2}
$$

i.e., even at heights exceeding the ion gyroradius (the result was derived in [4] under the stated assumption that $o_{<}<$). At even greater heights, when this inequality breaks down, the ion gyrocircles become siuall compared with the size of the illuminated mountain top, and the description of the gyrocircies as point particles moving strictly along the magnetic field becomes possible. This latter case was studied in detail in Ref. 4. It corresponds to the case considered at the beginning of this section, but with $\beta=\alpha$. Accordingly, the fraction of the wetted surface was found to be:

$$
\varepsilon_{i} \sim \alpha
$$

\subsection{Electron absorption}

At equal electron and ion temperatures, the electron gyroradii are much smaller than the ion gyroradii. Therefore, in a number of cases of practical interest the electron absorption corresponds to the regime of an infinitesimal gyrocircle defined by inequality

$$
h>\rho_{e} / \alpha^{1 / 2}
$$

In this regime, the fraction of the surface accessible to the electrons is determined by expression (11). In some cases, the regime defined by the inequality opposite to (12) may be realized. In such a regime the fraction of the surface accessible for electrons is determined from the estimate (9), bui with $\rho_{i}$ replaced by $\rho_{e}$. The division of the parameter space by these inequalities is shown in Fig. 3. Expressions for the fraction of the wetted surface are presented in the Table 1.

\section{Quasineutrality constraint}

\subsection{Potential structure near the wall}

We have been so far ignoring the presence of the electric fields and the quasineutrality constraint. The first effect of this constraint is that the bulk plasma must be charged positively with respect to the wall, to provide equal electron and ion currents (for definiteness, we consider the situation where there is no current to the wall). The corresponding potential is of the order of (3-5)T/e. For

$$
\alpha>\sqrt{m_{e} / m_{i}},
$$


a considerable fraction of this potential drop occurs within the Debye sheath immediately adjacent to the wall, whereas the rest of the drop occurs in a smooth fashion at distances of the order of the ion gyroradius (see, e.g., [1]). We will assume that inequality (13) is satisfied.

The second effect of the quasineutrality constraint is that, as we see from Table 1 and Fig. 4, in most regimes, if potentials are neglected, ions penetrate deeper beneath the mountain tops than the electrons (which are much more tightly tied to the magnetic field lines). Hence we see the second effect of the quasineutrality constraint: potentials must form to prevent ions from entering the spatial domain inaccessible for electrons in the shadows of the mountain tops. Therefore, with the quasineutrality constraint imposed, the area wetted by the ions will become equal to the area wetted by the electrons.

Table 1. Summary of wetted area vs. regime

\begin{tabular}{|c|c|c|}
\hline Domain & $\begin{array}{c}\text { Fraction of } \\
\text { surface wetted by } \\
\text { ions }\end{array}$ & $\begin{array}{c}\text { Fraction of } \\
\text { surface wetted } \\
\text { by electrons }\end{array}$ \\
\hline I & $\alpha$ & $\alpha$ \\
II & $\alpha^{4 / 5}\left(\rho_{i} / h\right)^{2 / 5}$ & $\alpha$ \\
III & $\alpha^{I / 2}$ & $\alpha$ \\
IV & $\alpha^{4 / 5}\left(\rho_{i} / h\right)^{2 / 5}$ & $\alpha^{4 / 5}\left(\rho_{e} / h\right)^{2 / 5}$ \\
V & $\alpha^{I / 2}$ & $\alpha^{4 / 5}\left(\rho_{e} / h\right)^{2 / 5}$ \\
\hline
\end{tabular}

When surface non-uniformities become smaller than the Debye radius, the ions entering the Debye sheath experience a strong acceleration in the normal direction to the wall and, because of this, approach the wall at an angle $\sim 1$. This restores uniform wetting of the surface by the ions and sets the applicability limit for the estimate (6). It is interesting to note that the plasma electrons in the case of the non-uniformities with $h<\rho_{e}$ still reach the wall only near the mountain tops, and the fraction of the area wetted by the electrons follows Eq. (6) even for the infinitesimally small cones.

\subsection{Ion reflection from the wall}

Consider for the moment a surface that does not absorb the ions at all. As was shown in Sec. 2, at $\alpha<1$, a typical ion coming from the plasma interior hits the wall at a shallow angle $\sim \alpha^{1 / 2}$, and starts "hopping" over the surface. Such an ion experiences a large number $N \sim 1 / \alpha$ bounces before it returns to the plasma (Cf. [13]). The absorption of the ions occurs only near the tips of the "mountains" (Fig. 1) accessible to the electrons. Obviously, a significant ion reflectivity will take place if the electron-absorbing fraction of the surface $\varepsilon_{e}$ is comparable to or less than $1 / N \sim \alpha$. This takes place in the domains II and III in Fig. 4. The domain I (where formally $\varepsilon_{e} \sim \alpha$ ) is of little interest because both electrons and ions in it are strongly magnetized, $\rho_{i}<<$, so that the ions hit the same zones at the mountain tops as the electrons, $\varepsilon_{i}=\varepsilon_{e}$. Accordingly, no significant ion reflection occurs in the domain 1. 
The ion albedo, $A$, in the zones II and III is of the order of 1 but not very close to one: $1-A \sim 1$. Indeed, the fraction $\varepsilon_{e}$ in these two zones is only of the order of $1 / N$, not much less than $I / N$. In the zones $I V$ and $V, \varepsilon_{e}$ is greater than $I / N$ and, therefore, the albedo is much less than 1 .

\subsection{Boundary conditions for the bulk plasma}

We will discuss the boundary conditions for plasma motion at a scale much greater than the spatial scale of the surface structures $h$, assuming that we are formulating them at large enough distance from the wall, where the presence of the fine features has already been smeared out. For the case of a significant ion albedo $A$, the relation between the density $n$ of the incoming plasma and the ion flux $q$ per unit surface element (comprising, as we have mentioned, a large number of bumps and dips) is:

$$
q=C_{q} \frac{\alpha n v_{T i}}{1+A}
$$

where $C_{q}$ is a numerical factor of the order of 1 depending on the details of the distribution function. Obviousiy, for the same particle source upstream and thereby the same particle Iux to the wall, the steady-state plasma density is higher at $A \sim 1$ than for a perfectly absorbing wall $(A=0)$. The way of using this hydrodynamic boundary condition for determining the flow parameters for the given sources is discussed, e.g., in Ref. [6]. For fluxtubes of varying cross-section and for the sources distributed along the field line there may exist a sonic transition, whose presence may require a supersonic flow at the wall. For the wall with a high albedo, this is impossible and may cause the appearance of the shock wave at some distance from the wall. In the expression (14) we have neglected the contribution for ExB drift which in some cases may become important $[1,14,15]$.

Consider now the sheath current-voltage characteristics. We denote the plasma

potential at a large distance from the wall as $\varphi$, which is positive to maintain the plasma neutrality. Then, the normal projection of the current density can be presented as:

$$
j_{n}=\operatorname{aenv}_{T i}\left(\sqrt{\frac{m_{i}}{2 \pi m_{e}}} \exp \left(-\frac{e \varphi}{T}\right)-\frac{C_{q}}{1+A}\right)
$$

One sees that the floating plasma potential

$$
\varphi=\frac{T}{e} \ln \frac{C_{q} \sqrt{m_{i} / 2 \pi m_{e}}}{1+A}
$$

(corresponding to a zero current to the wali) decreases for a finite albedo.

\section{Neutralization processes}

In this section we consider the dynamics of the ionization processes which may lead to generation of a cold neutralizing plasma in the shadowed zones. The plasma ions hitting the boundary of the domain in accessible for the electrons are reflected back to the plasma by the positive space-charge potential that is formed near the boundary (Fig. 2). For a small Debye length, the thickness of the transitional layer is, roughly speaking, the electron gyroradius. The material surfaces limiting the segment of the magnetic field line between two peaks are grounded (we consider a conducting wall). Therefore, the potential distribution along the magnetic field line in the reflecting layer (dotted line in Fig. 2 has the form shown in Fig. 2b. If electrons are formed by some process in the reflecting layer, they are trapped in this potential well. With this process continuing, the zone accessible to the ions will 
cventually rach the depth corresponding to the "geometrically" accessible level. Below we analyse two mechanisms that we consider most plausible neutralization mechanisms.

\subsection{Classical diffusion}

We are interested in the process by which electrons can penetrate into a magnetic field to the distance of the order of the ion gyro-radius or less (but grcater than the clectron gyro-radius). This is not quite a classical plasma diffusion, so we use this term here in a somewhat loose sense. Electrons experience scattering on the ions and, with every $90^{\circ}$ scattering penetrate by another distance $\sim \rho_{e}$ into the shadowed region; they don't get absorbed by the end-surfaces (Fig. 2) because of the presence of electrostatic confinement potential. To penetrate by the distance $\delta h_{i}$, they have to experience $\sim\left(\delta h_{i} / \rho_{e}\right)^{2}$ collisions with the ions (by collision we mean a $90^{\circ}$ scattering). This requires the time

$$
\tau_{D}^{(\text {class })} \sim \frac{\lambda_{C}}{v_{T e}}\left(\frac{\delta h_{i}}{\rho_{e}}\right)^{2}
$$

For $\delta h_{\Gamma} \sim \rho_{p}$, and a deuterium plasma with a temperature of $T_{e} \sim T_{i} \equiv T \sim 50 \mathrm{eV}$, density $n \sim 5 \cdot 10^{13}$ and the magnetic field of $3 T$, one has $\tau_{D}^{(a n o m)}, \sim 10 \mathrm{~ms}$.

\subsection{Anomalous diffusion}

The relative motion of the electrons with respect to the ions in the transitional layer may cause development of the lower-hybrid instability [16]. Initially, when the transitional layer is thin, of the order of the electron gyroradius, the relative velocity of the electrons and the ions is of the order of the electron thermal velocity, and the instability is very fast. However, as the transitional region broadens and reaches its final steady-state thickness, the instability slows down. Accordingly, the neutralization time is determined by this late stage of the instability, which we will discuss here.

For the thickness of the transitional layer $\sim \delta h_{i}$, the relative velocity of the electrons and ions is

$$
u \sim v_{T e} \frac{\rho_{e}}{\delta h_{i}}
$$

The nonlinear stage of the instability was considered in Refs. [17-20]. An estimate of the effective electron scattering frequency can be presented as:

$$
v_{e f f} \sim \eta \omega_{L H}\left(\frac{u}{v_{T i}}\right)^{2}
$$

where $\eta$ is a numerical factor whose value varies significantly from one analysis to another. We take the smallest value for $\boldsymbol{\eta}$ to obtain the upper-bound estimate for the diffusion time, $\eta \sim 10^{-2}$. The diffusion time is

$$
\tau_{D}^{(\text {anom })} \sim \frac{1}{v_{e f f}}\left(\frac{\delta h_{i}}{\rho_{e}}\right)^{2} \sim \frac{1}{\eta \omega_{C i}} \sqrt{\frac{m_{i}}{m_{e}}}\left(\frac{\delta h_{i}}{\rho_{i}}\right)^{4}
$$

Taking for a numerical estimate a point where $\delta h_{\Gamma} \sim \rho_{i}$, one finds that $\tau_{\text {Diff }}^{(\text {anom })} \sim 10^{4} / \omega_{C i}$. For a deuterium plasma in a magnetic field of $3 \mathrm{~T}$, this is $\sim 0.1 \mathrm{~ms}$.

Although the anomalous diffusion seems to be the fastest neutralization mechanisn, it may in fact be suppressed because of specific conditions of the problem, in particular, a 
small length of the fluxtube between two conducting surfaces, and a rapid variation in the direction of the drift velocity caused by the roughness of the neutralization boundary.

\section{Non-steady-state boundary conditions}

The presence of a new time scale related to neutralization processes causes the change of the boundary conditions between the slow and fast processes. For the slow processes, with the time-scale much longer than the neutralization time-scale $\tau$, one should use a "slow" form of the current-voltage characteristics, with the ion albedo $\mathrm{A}=0$ :

$$
j=j_{s} \text {, }
$$

with "s"="slow." For the fast process, with the time-scale much shorter than the neutralization time, the ion albedo is finite, and

$$
j=j_{f}
$$

with "f"="fast." To interpolate between the two situation, we suggest using the following boundary condition:

$$
\tau \frac{d}{d t}\left(j-j_{f}\right)=-\left(j-j_{s}\right)
$$

If plasma parameters vary slowly, one can neglecî the right-hand-side, and recover the steady boundary condition (21). If, on the other hand, plasma parameters are varying quickly, the left-hand-side dominates, and one recovers the boundary condition for unneutralized shadows. The same type of the boundary condition works for the particle flux:

$$
\tau \frac{d}{d t}\left(q-q_{f}\right)=-\left(q-q_{s}\right)
$$

\section{Secondary electron emission.}

It is well known that the secondary emission coefficient for a flat surface in a strongly tilted magnetic field may become much smaller than for a magnetic field intersccting the wall at an angle $\sim 1$ (see, e.g., [21] and references therein). Consider as an example a problem where the electron gyroradius is considerably smaller than the Debye radius and one can, therefore, neglect the effects of a normal electric field in the immcdiate vicinity of the surface. Then, the majority of the secondary electrons emitted from the wall will return to the wall because of their

gyro-motion. Only those secondary electrons whose velocity is directed almost parallel to the magnetic field will reach the bulk plasma. An estimate of the secondary emission coefficient $S$ reads in this case:

$$
S \sim \alpha{ }^{1 / 2} S_{0}
$$

(where $S_{0}$ is a secondary emission coefficient in the absence of the magnetic field, and the exact numerical facior depends on the angular distribution of the secondary electrons). In the case of a very strong magnetic field, where the electrons are strictly attached to field lines, transition to a rough surface would bring $S$ close to $S_{0}$, because the magnetic field intersects the surface of the cones at an angle $\sim 1$. At weaker magnetic fields, $S$ will tend to be smaller, because the surface wetted by relatively fast plasma electrons [satisfying condition (10)] would extend to a considerable distance beneath the mountain tips; hence the cold secondary electrons, which follow the field lines, will most often hit other mountain tops on their way from the surface. 


\section{Summary}

We have shown that the presence of a surface roughness may lead to a number of cffects directly influencing the physics of the divertor plasma. In particular, a significant ion albedo may appear for fast-enough perturbations of the incoming plasma flow. This affects both the current-voltage characteristics and the hydrodynamic boundary conditions and should be properly taken into account in the analysis of the non-steady-state processes near the wall. A significant change of the sccondary electron emission compared to the flat wall also affects the current-voltage charascteristics. On the other end of the problem is effect of the surface roughncss on the distribution of the heat and particle fluxes over the surface. In a broad range of parameters, a fraction of a wetted surface becomes significantly smaller than for a flat wall, giving rise to a strong local increase of the fluxes.

\section{Acknowledgment}

This work was performed for the U.S. Department of Energy by Lawrence Livermere National Laboratory under Contract W-7405-ENG-48 


\section{References}

1. R.H. Cohen, D.D. Ryutov. Phys. Plasmas, 2, p.2011 (1995).

2. D.D. Ryutov. Contrib. Plasma Phys., 36, 207 (1996).

3. R.H. Cohen, D.D. Ryutov. Nuclear Fusion, 37, p. 621 (1997).

4. R.H. Cohen, D.D. Ryutov. Phys. Plasmas, 5, p. 2194 (1998).

5. R.H. Cohen, R.E. Cid, E.B. Hooper, A.W. Molvik, G.D. Porter, and D.D. Ryutov Journ. of Nucl. Material, 266-269, 258 (1999).

6. R.H. Cohen, D.D. Ryutov. Phys. Plasmas, 6, 1995 (1999).

7. G.D. Porter, R.E. Cid, R.H. Cohen, E.B. Hooper, N. Luhmann, A.W. Molvik, and D.D. Ryutov. American Geophysical Union Transactions, 79, p. F675 (1998).

8. E. Deksnis, A. Cheetham, A. Hwang, P. Lonià, M. Pick, D.D.R. Summers, J.' Nuci. Mater. 176-177, 583 (1990).

9. J. Linke, H. Bolt, R. Doemer et al. J. Nucl. Mater. 176-177 856 (1990).

10. N. Yoshida, K. Tokunaga, T. Fujiwara, K. Tawara, T. Muroga, S. Itoh, J. Nucl. Mater. 196-198, 415(1992).

11. T. Tanabe, V. Philipps, K. Nakamura et al., J. Nucl. Mater. 241-243, 1164 (1997).

12. R.H. Cohen, D.D. Ryutov. Phys. Plasmas, 5, 973 (1998).

13. R.H. Cohen, D.D. Ryutov. Physics of Plasmas, 2, p. 4118 (1995).

14. D.Farina and R.Pozzoli, and D. Ryutov. Plasma Phys. Contr. Fus., 351271 (1993).

15. A.V. Chankin, P.C. Stangeby. Plasma Phys. Controlled Fusion, 36, 1485 (1994).

16. N.A. Krall, P.C. Liewer. Phys. Rev., A4, 2094 (1971).

17. J.F. Drake, P.N. Guzdar, J.D. Huba (1983). Phys, Fluids, 26, 601, (1983).

18. J.F. Drake, J.D. Huba, N.T. Gladd. Phys. Fluids, 26, 2247 (1983).

19. J.F. Drake, P.N. Guzdar, A.B. Hassam, J.D. Huba. Phys. Fluids, 27, 1148 (1984),

20. J.U. Brackbill, D.W. Forslund, K.B. Quest, and D. Winske, Phys. Fluids, 27, 2682 (1984).

21. S. Mizoshita, K. Shiraishi, N. Ohno, S. Takamura, J. Nucl. Mater., 220-222, 488 (1995). 


\section{Figure captions.}

Fig. 1 A rough surface made of randomly distributed cones of the same height $h$. The arrow shows the direction of the magnetic field; in the divertor geometry, the axis $x$ corresponds to the radial, the axis $y$ to the poloidal, and the axis $z$ to the toroidal directions.

Fig. 2 Formation of the zone inaccessible for the plasma ions (a). Potential distribution along the dotted line before the neutralization has began (b).

Fig. 3 The ion gyro-circle viewed in the direction of the magnetic field; the solid lines correspond to the instant when the lowest part of the circle is at the level of the mountain tops; the dashed lines correspond to some instant later in time, when the scraping-off of the ions begins.

Fig. 4 The parameter domain of the problem. The lines correspond to deuterium. The line $\alpha=\left(h / \rho_{e}\right)^{4 / 3}$ is not shown because it does not fit the scale. 


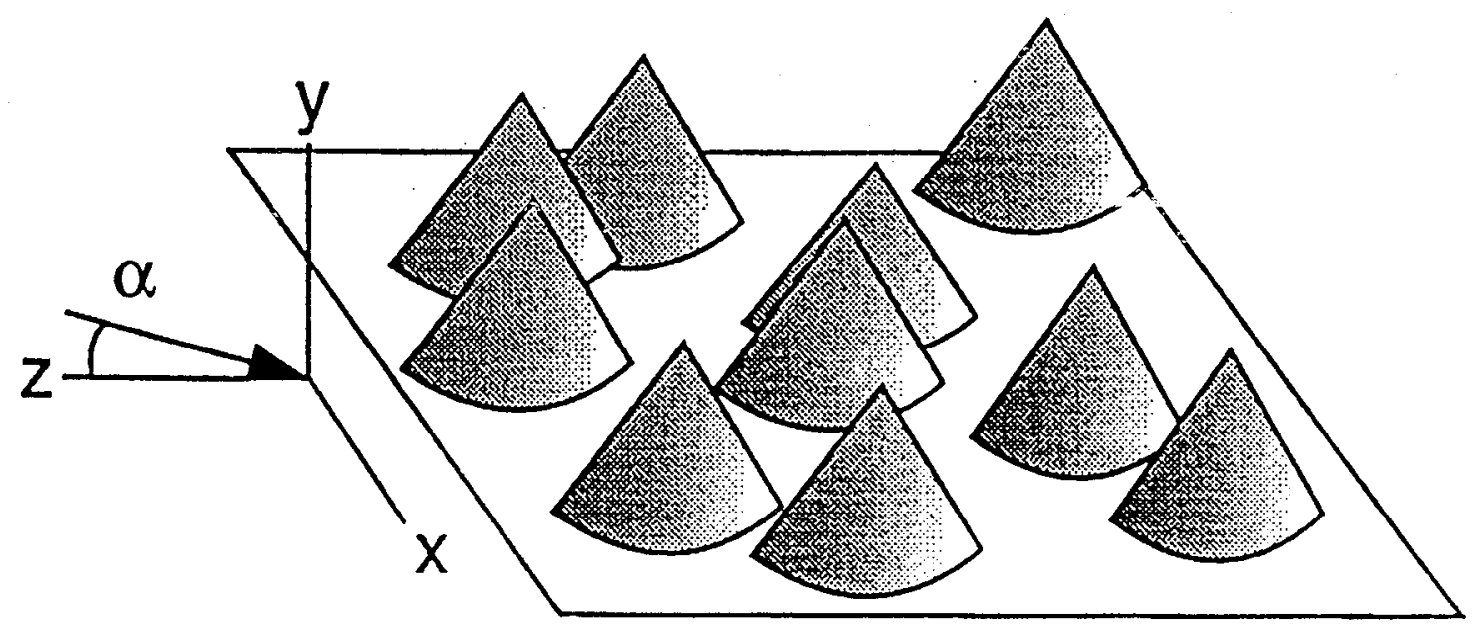

Fig. 1 
a)
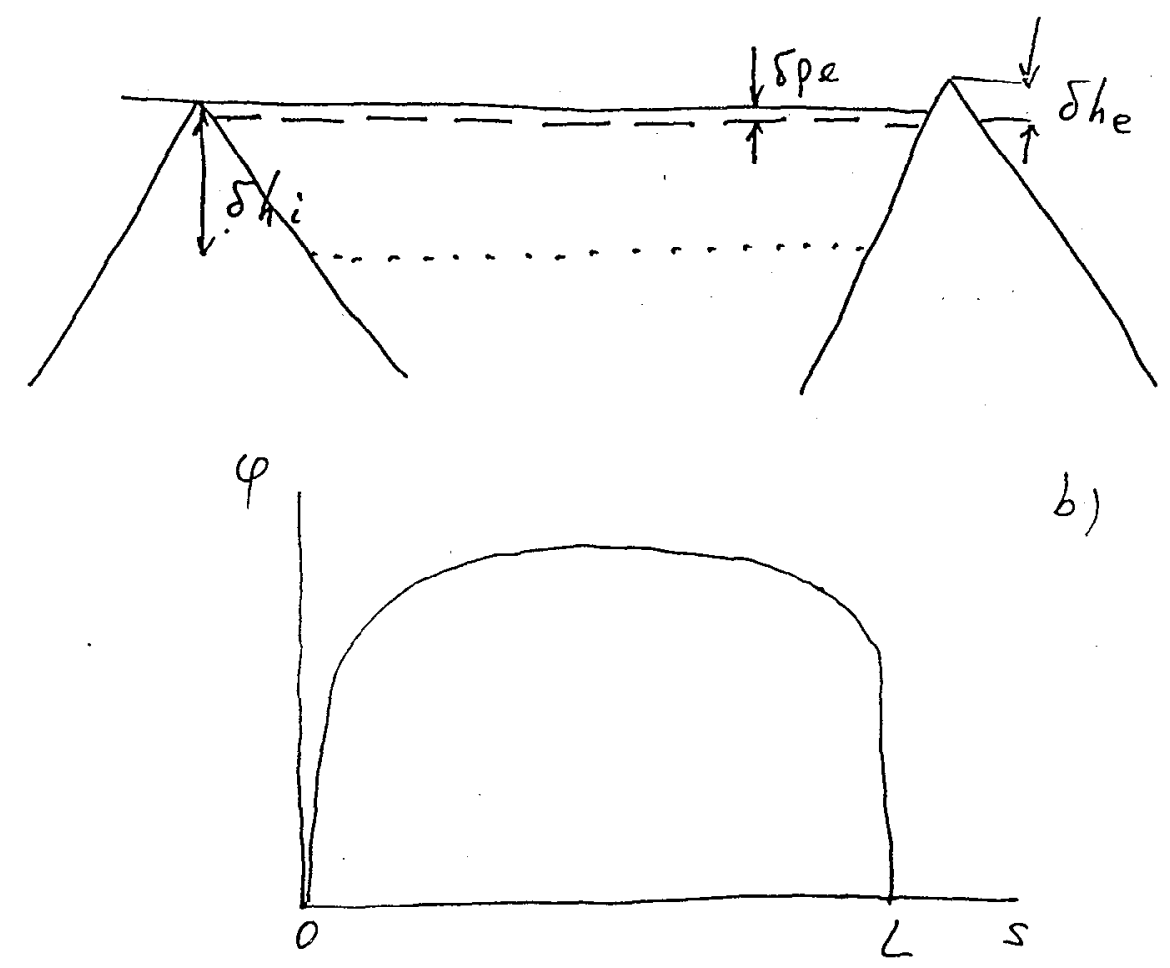

b)

Fig. 2 


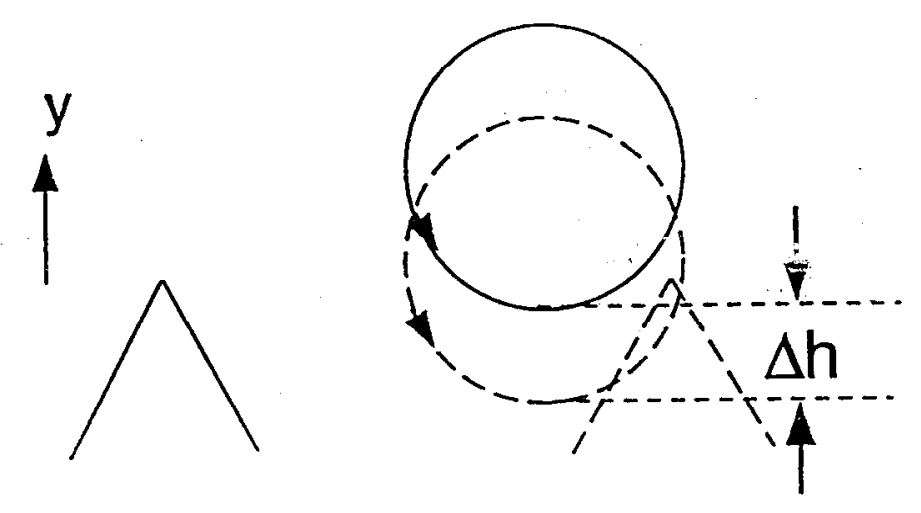

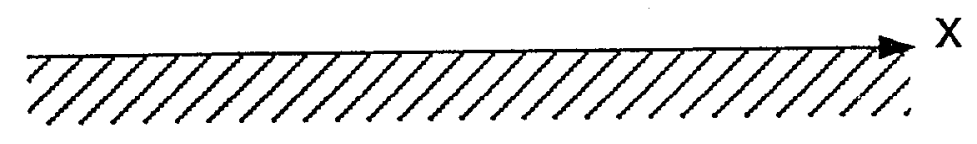

Fig. 3 


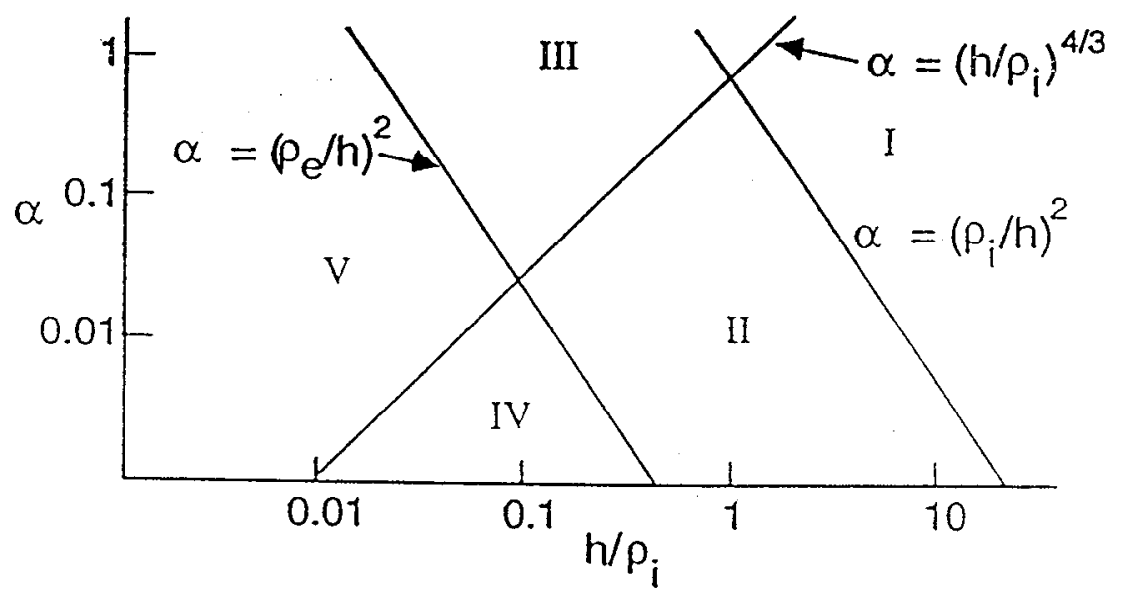

Fig. 4 